\title{
Der gute Arzt, oder über seine Proprien und Akzidenzien
}

\section{Markus Gassner}

Dr. med., Landarzt, Grabs

Kranke Menschen wünschen sich einen guten Arzt. Das ist begreiflich. Aber wie soll er denn sein, der gute Arzt? Manche möchten einen guten Menschen, am liebsten mit den besten Eigenschaften, mit der besten Qualität. Doch was bedeutet hier gut? Sind es Charaktereigenschaften oder andere Qualitätsmerkmale wie Fertigkeiten für eine kostengünstige Reparatur? Wer misst oder qualifiziert welche Qualität?

Bei den Dingen ist es einfacher. In der scholastischen Philosophie, die wir als Gymnasiasten noch lernen mussten, war es logisch, zwischen einem Proprium und einem Akzidenz zu unterscheiden. Ein Proprium umschreibt die wesentliche Eigenschaft, den Zweck, zum Beispiel eines Werkzeuges, die dazu dienen, Werke zu erzeugen.

\section{Das gute Messer}

Das Proprium eines Messers ist das Schneiden, das Teilen. Je nach Materie eignet sich nicht jedes Messer gleich gut bei der Zubereitung zum Essen von Brot, Gemüse, Fleisch oder Fisch. Auch hat jedes Messer ein unterschiedliches Risiko von Verletzungen, manche sind sogar dazu geschaffen (Säbel, Dolche). Man kann vor unbeabsichtigtem Schaden schützen oder einen Missbrauch über Gesetze behindern. Scharfrichter benutzten das Schwert, welches der Arzt Joseph-Ignace Guillotin aus humanitären Überlegungen perfektionierte.

Als Waffe wurden die vielen modifizierten Messer ersetzt. Die Polizei erhielten als Gendarmen (gens d'armes) die Lizenz, eine Waffe zu benutzen, oder als Carabinieri einen Karabiner zu tragen. Dort aufgepflanzte Messer (Bajonette) wurden wegen Maschinengewehren zu symbolischen zweckentfremdeten Insignien der Offiziere oder kantonal zu Stimmausweisen (Landsgemeindesäbel).

Jedes Messer hat seine Akzidenzien (zufällige Merkmale). Davon lebt das Marketing, es prägt Mode und Luxus, Angebot und Nachfrage. In der heutigen Zeit ist das Akzidenz aber oft wichtiger geworden als das Proprium: Gefälligkeit statt Wirksamkeit.

Bei Dingen ist der Unterschied einfacher messbar als bei Personen.

\section{Der gute Bundesrat}

Die besten Menschen sollten eigentlich ein Land führen. Doch nach welchen Eigenschaften wählen unsere Volksvertreter die besten Landesväter? Was ist ein Proprium, was ein Akzidenz eines guten Bundesrates? Nach welchen menschlichen Kriterien soll ein Land regiert werden?

Natürlich könnte man auch die wählenden Parlamentarier nach ihrer Qualität hinterfragen. Ihr Proprium wäre das Wohl der Eidgenossenschaft, das Akzidenz die Vertretung ihrer Partikularinteressen, die Partei, die Farben ihrer Wähler. Sie sollten die Besten für eine Aufgabe wählen und Gesetze zum Wohle aller Eidgenossen (inkl. Einwohner anderer Nationen, beispielsweise Touristen, Flüchtlinge usw.) gestalten, die Finanzierung dieser Aufgaben "steuern» im Sinne der Solidarität, auch der Gesundheit für Alle.

\section{Die Eigenschaften des guten Arztes}

Es gibt verschiedene Ärzte für zunehmend unterschiedliche Aufgaben. Sie benutzen für ihre Fertigkeiten unterschiedliche Werkzeuge. Wenige Neurochirurgen arbeiten mit speziellen, sehr teuren «Messern» (Gamma-Knife), nur auf Augen spezialisierte Ärzte sind häufiger und benutzen sehr kleine. Kosmetisch orientierte Ärzte befriedigen Kundenwünsche mit und ohne Messer. Pathologen, die sich nur um Verbrechen kümmern (Gerichtsmediziner) sollten keine Kundenwünsche erfüllen, arbeiten heute häufig messerlos.

Ärzte haben unterschiedliche persönliche Eigenschaften (Proprien) und arbeiten mit unterschiedlichen Möglichkeiten wie Lizenzen für unterschiedliche Messer, Gifte und Macht, mitunter aufgrund individueller Akzidenzien. Gibt es jedoch allgemeine Charaktereigenschaften eines guten Arztes? Hippokrates empfahl jungen Ärzten, sich Kenntnisse des Lebensstils, der Vegetation, des Bodens und Wassers zu verschaffen. Die Sprache der Kranken zu verstehen, war eine Selbstverständlichkeit.

Für die Qualität der Umwelt ist in der Natur die Artenvielfalt ein optimales Kriterium. Dies sollte auch für so komplexe Systeme wie «Gesundheit und Medizin» 


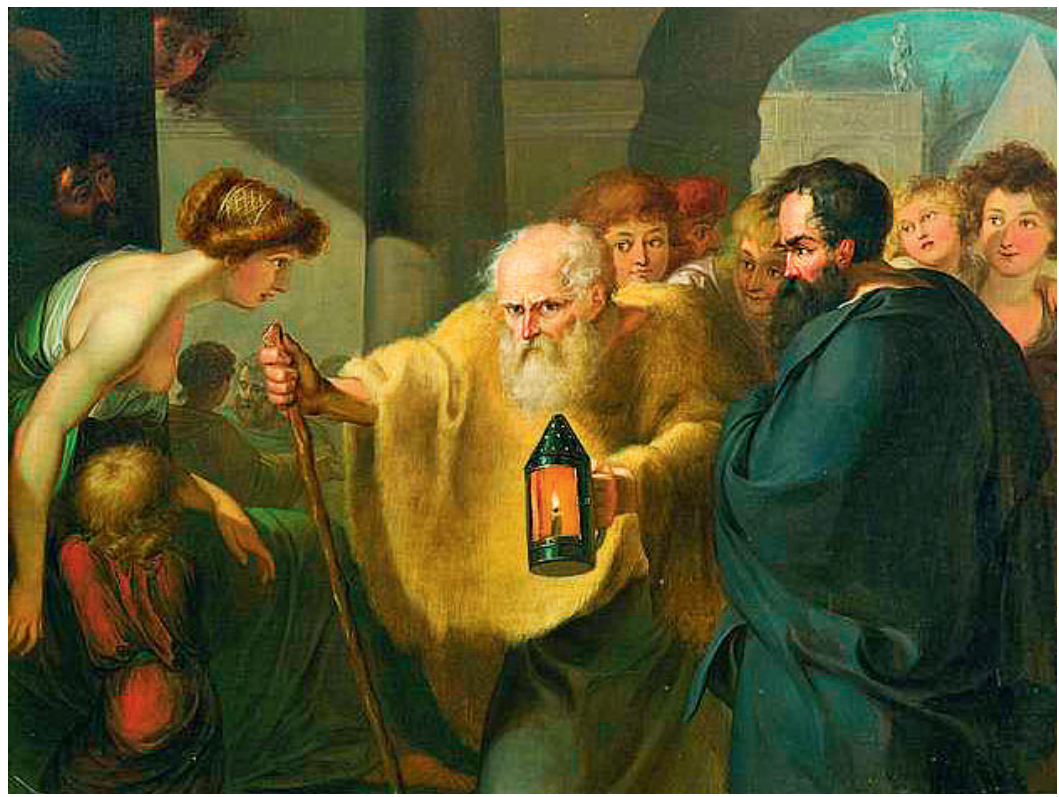

«Diogenes sucht einen Menschen» (Darstellung wahrscheinlich um 1780 von Johann Heinrich Wilhelm Tischbein) - suchte er vielleicht einen guten Arzt?

gelten. Jeder Arzt bearbeitet individuell ein kleines Biotop der Gesundheitsversorgung, meist über die Pflege von Patienten. Dies ändert sich kontinuierlich regional, über spezialisierte Angebote, aber auch schon rein physiologisch durch das Alter. Je älter ein Arzt wird, desto mehr behandelt er auch ältere, und somit polymorbide Menschen. Je nach Situation, eigener Ausbildung, und eigenem Interesse und Möglichkeiten wird er Patienten neu übernehmen, für technische Leistungen weiterweisen oder rechtzeitig Kollegen um Rat fragen. Dieser Dialog bleibt immer noch die beste Weiterbildung.

\section{Wie findet man seinen guten Arzt?}

Kranke Schweizer möchten gute Ärzte, und ihren Arzt selbst auswählen. Dazu müssen sie seit jeher Kompromisse eingehen. Früher hatten die meisten Familien ihren Hausarzt und ihre Krankenkasse. Nicht nur soziale Probleme, auch Notfälle wurden so einfach und kostengünstig gelöst. Die Schweizerische Akademie der Medizinischen Wissenschaften hatte 2001 Bedürfnisse und Lösungen bei Notfällen in der Schweiz untersucht [1]. In den letzten 15 Jahren haben sich hier sowohl Angebot wie Nachfrage wesentlich verändert. Ein Arzt ist nicht für jedes aktuelle gesundheitliche Problem für jeden gleich erreichbar, wie eine Schweizer Klinik für einen Scheich nach einem Skiunfall. Schweizer suchen an einem Donnerstag wegen einem akuten Bibeli mitunter vergebens ihren Arzt mit dem optimalsten Rating und Unterstützung per Telmed oder Medcal. Passiert doch etwas ernsthaftes Ende Dezem- ber, ist ihre doppelte, hohe Franchise trotz Empfehlung von Comparis dahin. Gesunde sind begeistert und wechseln Kassen, Kranke wollen selten Managed Care. Sie möchten keine «Kunden» sein.

Manche gesunde und kranke Menschen wünschen keine Spitzenmedizin, sie leben freiwillig oder genötigt bescheiden und erinnern an den in einem Fass lebenden Diogenes (wörtlich «der von Gott Gezeugte»). Seinen König schickt er weg, weil er ihm vor der Sonne steht, zynisch konsequent: Die Menschen brauchen nur das Notwendigste. Mit einer Lampe sucht er mittags auf dem Markt aber «einen Menschen», vielleicht seinen Arzt!

Mit zunehmendem Alter und Erfahrung stellen wir fest, wie jedes zusätzliche Wissen die Welt des Nichtwissens vergrössert. Solange wir denken können, sollte dies die Gier nach Neuem wecken. Entdecken ist köstlicher als konsumieren! Forschen in einer Landpraxis ist so einfach. Es gibt hierzu neue, sehr hoffnungsvolle Ansätze. Multimorbide und betagte Patienten relativieren heute das Denken nach Guidelines und Trampeln auf Patientenpfaden! Das Vertrauen in Lehrmeinungen ist erschüttert, eine gute Chance, diese jetzt zu hinterfragen und neues Wissen zu suchen Welchen Arzt wollen Sie als Patient? Welche Eigenschaften soll Ihr Arzt heute und in den nächsten Jahren haben? Wie viel ist Ihnen dies wert? Wer soll diese Eigenschaften (Qualität!) bewerten, bestimmen, messen, fördern? Wer wählt wie die besten Studenten aus, für das Medizinstudium?

Eines ist gewiss, neoliberale marktwirtschaftliche Überlegungen haben bisher keine sozialen Probleme weltweit und auch in kleineren Gemeinschaften langfristig gelöst, viele aber verstärkt. Was und welche Arbeit wie viel wert ist, hat sich während der letzten Dekaden merklich verändert [2, 3]. Wissenschaftlich sollten Ärzte heute wieder etwas mehr philosophisch denken, auch mit mehr Liebe zur Weisheit, neugierig, weniger rein analytisch monovalent, vor allem aber weniger administrativ gewinnorientiert, geldgierig. Umgekehrt müssen wir Ärzte uns auch mit allen politischen Mitteln wehren, nur ökonomisch bewertet und missbraucht zu werden

In der Schweiz (Demokratie: vom Volk für das Volk), die sich zusätzlich als Genossenschaft bezeichnet, sollten deshalb gute Charaktereigenschaften, Proprien und nicht vorwiegend Akzidenzien wie Fertigkeiten der Ärzte politisch gefördert werden. Jede Bevölkerung einer Demokratie hat die Regierung und die Ärzte, die sie verdient.

\section{Bildnachweis}

Diogenes sucht einen Menschen

- Wikipedia, gemeinfrei 